\title{
Significance of left ventricular apical-basal muscle bundle identified by cardiovascular magnetic resonance imaging in patients with hypertrophic
} cardiomyopathy

\author{
Christiane Gruner ${ }^{1,2 *}$, Raymond H. Chan ${ }^{3,4}$, Andrew Crean ${ }^{1}$, Harry Rakowski ${ }^{1}$, \\ Ethan J. Rowin ${ }^{5}$, Melanie Care ${ }^{6,7}$, Djeven Deva ${ }^{1}$, Lynne Williams ${ }^{1}$, Evan Appelbaum ${ }^{3,4}$, \\ C. Michael Gibson ${ }^{3,4}$, John R. Lesser ${ }^{8}$, Tammy S. Haas ${ }^{8}$, James E. Udelson ${ }^{5}$, \\ Warren J. Manning, ${ }^{3,4}$, Katherine Siminovitch ${ }^{6,7}$, Anthony C. Ralph-Edwards', \\ Hassan Rastegar ${ }^{5}$, Barry J. Maron ${ }^{8}$, and Martin S. Maron ${ }^{5}$
}

\begin{abstract}
${ }^{1}$ Division of Cardiology and Cardiovascular Surgery, Peter Munk Cardiac Centre, Toronto General Hospital, University Health Network, Toronto, ON, Canada; ${ }^{2}$ Division of Cardiology, Cardiovascular Center, University Hospital, Raemistrasse 100, 8091 Zurich, Switzerland; ${ }^{3}$ PERFUSE Core Laboratory and Data Coordinating Center, Harvard Medical School, Boston, MA, USA; ${ }^{4}$ Department of Medicine, Cardiovascular Division, Beth Israel Deaconess Medical Center, Harvard Medical School, Boston, MA, USA; ${ }^{5}$ Hypertrophic Cardiomyopathy Center, Division of Cardiology, Tufts Medical Center, Boston, MA, USA; ${ }^{6}$ Fred A. Litwin and Family Centre in Genetic Medicine, Mount Sinai Hospital \& University Health Network, Toronto, ON, Canada; ${ }^{7}$ Department of Medicine, University of Toronto and Samuel Lunenfeld and Toronto General Research Institutes, Toronto, ON, Canada; and ${ }^{8}$ Hypertrophic Cardiomyopathy Center, Minneapolis Heart Institute Foundation, Minneapolis, MN, USA
\end{abstract}

Received 8 September 2013; revised 20 January 2014; accepted 17 March 2014; online publish-ahead-of-print 8 May 2014

Aims

Cardiovascular magnetic resonance (CMR) has improved diagnostic and management strategies in hypertrophic cardiomyopathy $(\mathrm{HCM})$ by expanding our appreciation for the diverse phenotypic expression. We sought to characterize the prevalence and clinical significance of a recently identified accessory left ventricular (LV) muscle bundle extending from the apex to the basal septum or anterior wall (i.e. apical-basal).

Methods

and results

CMR was performed in 230 genotyped HCM patients ( $48 \pm 15$ years, $69 \%$ male), 30 genotype-positive/phenotype-negative $(G+/ P-)$ family members ( $32 \pm 15$ years, $30 \%$ male), and 126 controls. Left ventricular apical-basal muscle bundle was identified in 145 of 230 (63\%) HCM patients, 18 of 30 (60\%) G+/P- family members, and 12 of 126 (10\%) controls ( $\mathrm{G}+/ \mathrm{P}$ - vs. controls; $P<0.01$ ). In HCM patients, the prevalence of an apical-basal muscle bundle was similar among those with disease-causing sarcomere mutations compared with patients without mutation (64 vs. $62 \% ; P=0.88$ ). The presence of an LV apical-basal muscle bundle was not associated with LV outflow tract obstruction $(P=0.61)$. In follow-up, 33 patients underwent surgical myectomy of whom $22(67 \%)$ were identified to have an accessory LV apical-basal muscle bundle, which was resected in all patients.

Conclusion $\quad$ Apical-basal muscle bundles are a unique myocardial structure commonly present in $\mathrm{HCM}$ patients as well as in $\mathrm{G}+/ \mathrm{P}-$ family members and may represent an additional morphologic marker for HCM diagnosis in genotype-positive status.

Keywords

Hypertrophic cardiomyopathy • Cardiovascular magnetic resonance • Muscle bundle • Genotype

\section{Introduction}

Over the last decade, cardiovascular magnetic resonance (CMR) has emerged as an advanced imaging technique, which provides a comprehensive assessment of hypertrophic cardiomyopathy (HCM) morphology. ${ }^{1}$ With tomographic, high spatial resolution imaging, the unique imaging strengths of CMR have led to an expanded appreciation of the heterogeneous phenotypic expression

* Corresponding author. Tel: +414425513 69, Fax: +414425587 01, Email: christiane.gruner@gmx.ch; Christiane.Gruner@usz.ch

Published on behalf of the European Society of Cardiology. All rights reserved. (C) The Author 2014. For permissions please email: journals.permissions@oup.com 
of HCM beyond left ventricular (LV) hypertrophy, ${ }^{2}$ and mitral valve abnormalities $^{3-5}$ to include morphologic abnormalities of the right ventricle, ${ }^{6}$ papillary muscle architecture, ${ }^{7}$ and myocardial crypts ${ }^{8-10}$ as well as detection of fibrosis for risk assessment. ${ }^{11-13}$ By virtue of identifying these structural abnormalities, CMR has resolved diagnostic dilemmas, altered management strategies in patients undergoing invasive septal reduction therapies and enhanced risk assessment. ${ }^{13-15}$

With the growing penetration of CMR into the routine evaluation of $\mathrm{HCM}$ patients, we have observed unique and distinctive accessory LV muscle bundles, extending from the apex to the basal anterior septum or anterior free wall (i.e. apical-basal). Therefore, the aim of our study was to use CMR to characterize the prevalence and clinical significance of LV apical-basal muscle bundles among a large cohort of genotyped HCM patients, genotype-positive/phenotypenegative $(\mathrm{G}+/ \mathrm{P}-)$ family members, and healthy controls.

\section{Methods}

\section{Patient populations}

The study cohort included 230 consecutive genetically tested HCM patients who underwent CMR between 1 August 2004 and 30 November 2009 at three North American HCM tertiary referral centres (Tufts Medical Center, Boston, MA, USA; Minneapolis Heart Institute, Minneapolis, MN, USA; and Toronto General Hospital, Toronto, ON, Canada). The diagnosis of HCM was based on demonstration by CMR of a nondilated, hypertrophied LV (maximal wall thickness $\geq 15 \mathrm{~mm}$ ) in the absence of another cardiac or systemic disease that could produce the magnitude of hypertrophy evident. ${ }^{16}$ Patients in the end-stage phase of HCM (ejection fraction $\leq 50 \%$ ), previous alcohol septal ablation or surgical septal myectomy, history of prior defibrillator (ICD)/pacemaker implantation or other contraindications to CMR were excluded. In addition, no patient had confirmed anomalous insertion of anterolateral papillary muscle directly into anterior mitral leaflet. ${ }^{17,18}$ Clinical follow-up duration for study patients was from the time of initial assessment during which a CMR study was obtained, extending to the most recent evaluation ascertained in the clinic or by telephone interview as of 30 March 2013 (median follow-up time 3.5 years (interquartile range $2.5-4.8$ years).

In HCM patients, left ventricular outflow tract (LVOT) obstruction was defined by continuous-wave Doppler echocardiography as a peak instantaneous outflow gradient $\geq 30 \mathrm{mmHg}$ under resting conditions. During the follow-up period, patients who underwent surgical myectomy were identified and echocardiography, CMR, and the operative reports were reviewed to determine whether an accessory LV apical-basal muscle bundle was resected, as well as to clarify how the accessory muscle bundle may have contributed to the mechanism of LVOT obstruction. In all patients who underwent myectomy, LVOT gradients postmyectomy were derived from echocardiography performed at least 3 months from operative date.

In addition, $30 \mathrm{G}+/ \mathrm{P}$ - family members (age $32 \pm 15$ years; $30 \%$ male) were recruited at the Tufts Medical Center and the Toronto General Hospital. They were clinically asymptomatic and their maximal wall thickness was $\leq 12 \mathrm{~mm}$ (or normal adjusted to body surface area (BSA) and age), and no LVOT obstruction was present. During the same study period, 126 patients were assessed by clinical evaluation and CMR, without evidence of cardiovascular disease at the Minneapolis Heart Institute and at the Toronto General Hospital, and served as normal control subjects.

The study was approved by the Research Ethics Board of the University Health Network in Canada and by the Investigational Review Board of the participating US centres and it complies with the Declaration of Helsinki.
All authors had full access to the data, take full responsibility for its integrity, and have agreed to the manuscript as written.

\section{Cardiovascular magnetic resonance}

Images from all centres were transferred to a core laboratory (PERFUSE, Boston, MA, USA) for central, blinded analysis. Left ventricular volume, mass and ejection fraction were measured from short-axis datasets using standard volumetric techniques and analysed with commercially available software (QMASS ${ }^{\circledR}$ v7.4, Medis Inc., The Netherlands). Typical scan parameters for CMR cine imaging were as follows: standard ECG-gated, steady-state free precession (SSFP), voxel size $2.0 \times 0.5 \mathrm{~mm}$, TR/TE 39.6/1/12 ms, flip angle $55^{\circ}$, matrix $192 \times 192$, temporal resolution $=40 \mathrm{~ms}$. Three-chamber SSFP sequences were prescribed manually from basal short-axis views with multiple contiguous $8 \mathrm{~mm}$ thick slices with no interslice gaps to ensure full ventricular coverage.

The LV chamber was assessed according to American Heart Association 17 segment model. End-diastolic and end-systolic LV endocardial and epicardial borders were manually planimetered to define the myocardium, taking care to exclude papillary muscles and intertrabecular blood pool. Maximal LV wall thickness was defined as the greatest dimension at any site within the LV chamber on end-diastolic images. Left ventricular volumes and mass data were indexed to BSA.

For late gadolinium enhancement (LGE) imaging, image acquisition was performed $10-15 \mathrm{~min}$ after injection of $0.02 \mathrm{mmol} / \mathrm{kg}$ gadolinium contrast agent with 2D breath-held segmented inversion-recovery sequence, acquired in the same orientation as the cine SSFP images. Quantification of LGE was performed by manually adjusting a greyscale threshold to define areas of visually identified LGE. ${ }^{19}$ These areas were then summed to generate a total volume of LGE and expressed as a proportion of total LV mass myocardium (\%LGE).

An accessory LV muscle bundle was defined in either the 2- or 3-chamber long-axis cine image as a single band of muscle extending from apex through the LV cavity to the basal septum or anterior wall (i.e. apical-basal) without evidence of chordal attachment to the mitral valve (Figure 1).

The LV morphologic analysis was performed by one experienced reader, R.C. (with 3 years of CMR experience) and reviewed and confirmed by a second expert reader (E.A.; with 10 years of CMR experience). The LV apical-basal muscle bundle analysis was performed by C.G. (with 3 years of experience in CMR image interpretation) and these readings were reviewed and confirmed by a second expert reader (A.C.; with 15 years of CMR experience). Both these independent observers were blinded to patient identity and clinical profile.

\section{Mutational analysis}

Patients were screened with laboratory-specific methods for identification of mutations in the protein coding exons and intron/exon boundaries sequencing the eight myofilament genes which most commonly cause HCM, including: myosin-binding protein C (MYBPC3), $\beta$-myosin heavy chain (MYH7), essential and regulatory myosin light chains (MYL2, MYL3), cardiac troponin T (TNNT2), cardiac troponin I (TNNI3), $\alpha$-tropomyosin (TPM1), and cardiac actin (ACTC). A DNA variant was considered disease-causing based on the following criteria: (i) an amino acid change which is conserved among species and isoforms; (ii) the absence of the mutation in $>300$ unrelated chromosomes from healthy, ethnic-matched controls; (iii) co-segregation with affected members in the family; and (iv) previously reported to be disease-causing in HCM patients. Based on these criteria, the laboratory classified mutations as pathogenic, presumed pathogenic, variants of unknown significance or benign. For the purposes of this study, a pathogenic or presumed pathogenic mutation was considered a positive genotype. ${ }^{20}$ 

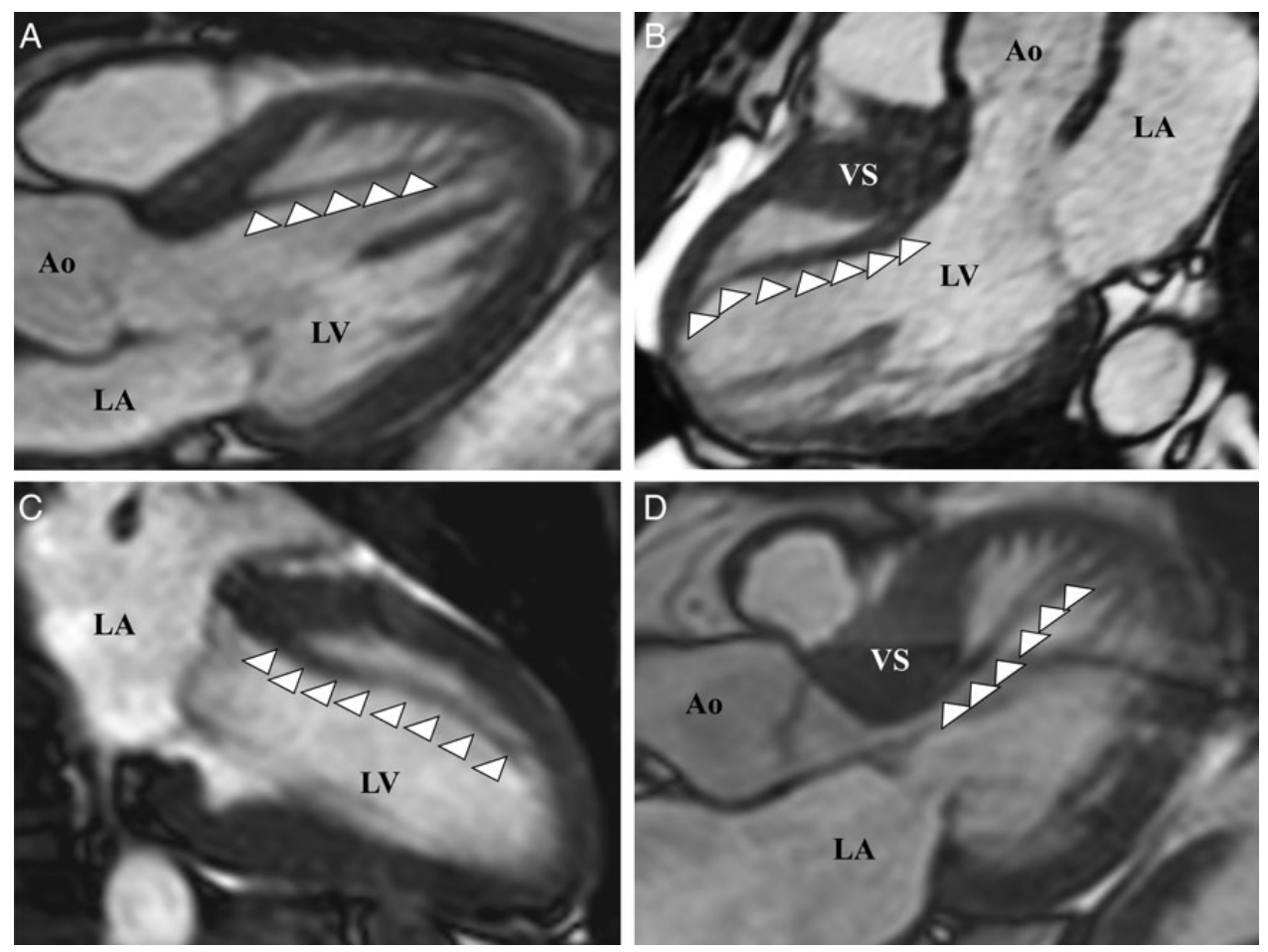

Figure I Spectrum of accessory left ventricular apical-basal muscle bundles in hypertrophic cardiomyopathy patients. (A) Accessory left ventricular apical muscle bundle (arrowheads) in asymptomatic obstructed 17-year-old hypertrophic cardiomyopathy patient with minimal left ventricular hypertrophy $(16 \mathrm{~mm})$. (B) A 64-year-old man with non-obstructive hypertrophic cardiomyopathy, maximal left ventricular wall thickness of $18 \mathrm{~mm}$ and genotyped to a MYBPC3 mutation (Trp792fs) with a prominent left ventricular apical-basal muscle bundle (arrowheads). (C) Asymptomatic 28-year-old female with non-obstructive hypertrophic cardiomyopathy with focal hypertrophy limited to the basal ventricular septum $(16 \mathrm{~mm})$ and an accessory left ventricular apical-basal muscle bundle (arrowheads) traversing the left ventricular cavity from the anterior wall to the distal portion of the chamber. (D) 33-year-old man with NYHA Class III symptoms due to hypertrophic cardiomyopathy with resting left ventricular outflow tract obstruction $(70 \mathrm{mmHg}$ ) and maximal left ventricular wall thickness of $24 \mathrm{~mm}$ with an left ventricular apical-basal muscle bundle (arrowheads). Following CMR evaluation, patient underwent surgical myectomy for relief of left ventricular outflow tract obstruction. Ao, aorta; LA, left atrium; LV, left ventricle; VS, ventricular septum.

\section{Reproducibility}

Inter- and intraobserver variability for the presence or absence of LV apical-basal muscle bundle was assessed from the CMR studies from the HCM cohort of 230 patients. For interobserver variability, two readers (A.C. and C.G.) independently assessed for the presence or absence of muscle bundle without prior knowledge of the clinical data, and were blinded to the previous results. For intraobserver variability, one reader (C.G.) independently assessed 50 randomly selected studies for the presence or absence muscle bundles in an identical fashion on two occasions (12 months apart), also blinded to the clinical data.

\section{Statistical analysis}

Continuous and categorical data are expressed as mean \pm SD or $n(\%)$, respectively. Comparisons of characteristics between groups were made with unpaired Student's $t$-test, one-way ANOVA, $\chi^{2}$ tests, or Fisher's exact test where appropriate for continuous and categorical data. Adjustments for multiple comparisons were made with Scheffe's method or Bonferroni correction where appropriate. Reproducibility was assessed by measure of agreements with $\kappa$ coefficient. A $P$-value of $<0.05$ was considered statistically significant.

\section{Results}

\section{Patient characteristics}

Clinical, demographic, and imaging findings for the $230 \mathrm{HCM}$ patients, $30 \mathrm{G}+/ \mathrm{P}$ - family members, and 126 normal controls are shown in Table 1. Hypertrophic cardiomyopathy patients were older and more often male compared with G+/P - family members. Genetic test results for $\mathrm{HCM}$ patients and $\mathrm{G}+/ \mathrm{P}$ - family members are given in Table 2. Among HCM patients, $32 \%$ were found to have a positive genotype, with mutations in the MYH7 and MYBPC3 genes accounting for $79 \%$ of disease-causing mutations.

\section{Characteristics of left ventricular apical-basal muscle bundle in hypertrophic cardiomyopathy patients}

An accessory LV apical-basal muscle bundle, identified by CMR, was present in 145 (63\%) HCM patients (Figure 1). There was no difference in the prevalence of an LV apical-basal muscle bundle among those HCM patients found to have a disease-causing sarcomere 
Table I Clinical characteristics and cardiovascular magnetic resonance findings in hypertrophic cardiomyopathy patients, $\mathbf{G}+/ \mathbf{P}-$ family members and normal controls

\begin{tabular}{|c|c|c|c|c|}
\hline & HCM patients & $\mathbf{G}+/ \mathbf{P}-$ family members & Normal controls & $P$-value* \\
\hline No. of patients & 230 & 30 & 126 & \\
\hline Age at CMR (years) & $48.4 \pm 15.1$ & $31.9 \pm 14.7$ & $40.9 \pm 16.6$ & $<0.0001$ \\
\hline Male, $n$ & $158(69 \%)$ & $9(30 \%)$ & $69(55 \%)$ & $<0.0001$ \\
\hline Body surface area $\left(\mathrm{g} / \mathrm{m}^{2}\right)$ & $1.96 \pm 0.24$ & $1.81 \pm 0.25$ & $1.93 \pm 0.26$ & $<0.009$ \\
\hline NYHA Class I/II/III/IV, n & $120 / 89 / 20 / 1$ & $30 / 0 / 0 / 0$ & $126 / 0 / 0 / 0$ & $<0.0001$ \\
\hline LVOT obstruction at rest ( $\geq 30 \mathrm{mmHg}), n$ & $45(20 \%)$ & 0 & 0 & $<0.0001$ \\
\hline Septal reduction therapy, $n$ & $37(16 \%)$ & 0 & 0 & $<0.0001$ \\
\hline LV apical-basal muscle bundle, $n$ & $145(63 \%)$ & $18(60 \%)$ & $12(10 \%)$ & $<0.0001$ \\
\hline LVEDV indexed to $B S A\left(\mathrm{~mL} / \mathrm{m}^{2}\right)$ & $77.5 \pm 17.1$ & $74.8 \pm 13.0$ & $85.3 \pm 15.3$ & $<0.0001$ \\
\hline Ejection fraction (\%) & $66.6 \pm 8.4$ & $64.9 \pm 4.4$ & $62.4 \pm 6.3$ & $<0.0001$ \\
\hline $\mathrm{LA}$ volume indexed to $\mathrm{BSA}\left(\mathrm{mL} / \mathrm{m}^{2}\right)$ & $57 \pm 23$ & $40 \pm 9$ & NA & $<0.0001$ \\
\hline Maximum LV wall thickness (mm) & $19.4 \pm 5$ & $9.7 \pm 1.5$ & $7.8 \pm 2.0$ & $<0.0001$ \\
\hline $\mathrm{LV}$ mass indexed to $\mathrm{BSA}\left(\mathrm{g} / \mathrm{m}^{2}\right)$ & $75.9 \pm 32.9$ & $42.7 \pm 9.6$ & $52.8 \pm 11.5$ & $<0.0001$ \\
\hline LGE present, $n$ & 95 (41.3\%) & NA & NA & NA \\
\hline LGE percentage (\%) & $20.3 \pm 14.5$ & NA & NA & NA \\
\hline Positive genotype, $n$ & $73(32 \%)$ & $30(100 \%)$ & NA & $<0.0001$ \\
\hline
\end{tabular}

CMR, cardiovascular magnetic resonance; HCM, hypertrophic cardiomyopathy; G+/P-, genotype positive/phenotype negative; NYHA, New York Heart Association; LGE, late gadolinium enhancement; LVOT, left ventricular outflow tract; LVEDV, left ventricular end-diastolic volume; LA, left atrium; LV, left ventricle.

*One $P$-value across all three subgroups.

Table 2 Distribution of sarcomere protein gene mutations in hypertrophic cardiomyopathy patients and $\mathbf{G}+/ \mathbf{P}-$ family members

\begin{tabular}{lll}
\hline $\begin{array}{l}\text { Sarcomere } \\
\text { mutations }\end{array}$ & $\begin{array}{l}\text { HCM patients } \\
(\mathbf{n}=\mathbf{7 3})\end{array}$ & $\begin{array}{l}\mathbf{G}+/ \mathbf{P}-\text { family } \\
\text { members }(\mathbf{n}=\mathbf{3 0})\end{array}$ \\
$\ldots \ldots \ldots \ldots \ldots \ldots \ldots \ldots \ldots \ldots \ldots \ldots \ldots \ldots \ldots \ldots \ldots \ldots \ldots \ldots \ldots \ldots \ldots \ldots \ldots \ldots \ldots \ldots \ldots \ldots \ldots \ldots \ldots \ldots \ldots$ \\
MYH7, $n(\%)$ & $35(48)$ & $7(23)$ \\
MYBPC3, $n(\%)$ & $23(32)$ & $15(50)$ \\
TNNT2, $n(\%)$ & $5(7)$ & $3(10)$ \\
TNNI3, $n(\%)$ & $3(4)$ & 0 \\
MYL2, $n(\%)$ & $1(1.4)$ & $2(7)$ \\
TPM1, $n(\%)$ & $2(2.8)$ & $1(3)$ \\
ACTC, $n(\%)$ & 0 & 0 \\
MYL3, $n(\%)$ & 0 & 0 \\
Multiple, $n(\%)$ & $4(5.5)$ & $2(7)$ \\
\hline
\end{tabular}

HCM, hypertrophic cardiomyopathy; G+/P-, genotype positive/phenotype negative; MYH7, $\beta$-myosin heavy chain; MYBPC 3 , myosin-binding protein $C$; TNNT2, cardiac troponin T; TNNI3, cardiac troponin I; MYL2 and 3, essential and regulatory myosin light chains; TPM1, $\alpha$-tropomyosin; ACTC, cardiac actin.

protein gene mutation (47 of $73 ; 64 \%$ ) compared with patients with no mutation identified ( 98 of $157 ; 62 \% ; P=0.88$ ).

A similar proportion of young $\mathrm{HCM}$ patients $<20$ years of age (7 of 12; 58\%) had a muscle bundle compared with HCM patients $>60$ years ( 35 of $51 ; 69 \% ; P=0.14$ ). In addition, the prevalence of an LV apical-basal muscle bundle was comparable across the range of maximal LV wall thickness measurements, including in 65 of 100 patients $(65 \%)$ with mild LV hypertrophy $(\leq 18 \mathrm{~mm})$ and in 18 of 26 (69\%) patients with marked LV hypertrophy $(\geq 25 \mathrm{~mm} ; P=$ $0.5)$. Patients with hypertrophy confined only to the apex or lateral wall also had a similar prevalence of LV apical-basal muscle bundles compared with those with hypertrophy confined to only the ventricular septum (apical 34 of $57 ; 60 \%$ vs. lateral wall 5 of 8 ; $63 \%$ vs. septal 106 of $165 ; 64 \%, P=0.8)$. There was no difference with respect to the presence of an LV apical-basal muscle bundle and a number of other relevant HCM disease-related variables including: gender $(P=0.66)$, left atrial volume indexed to BSA ( $P=$ 0.25), LV ejection fraction $(P=0.23)$, or New York Heart Association (NYHA) functional class $(P=0.32)$. LGE was present in the LV wall in $95 \mathrm{HCM}$ patients (41\%), although no patient had focal LGE involving the LV apical-basal muscle bundle.

\section{Left ventricular outflow tract obstruction and surgical myectomy}

There was no difference in the presence of LVOT obstruction $\geq 30 \mathrm{mmHg}$ among HCM patients with or without an LV apicalbasal muscle bundle (30 of $145 ; 21 \%$ vs. 15 of $85 ; 18 \% ; P=0.61$ ) nor was there an association in patients with obstruction between the magnitude of LVOT gradient and the presence of an apicalbasal muscle bundle $(P=0.44)$.

However, during the follow-up period, 33 patients underwent surgical myectomy for relief of LVOT obstruction, of which 22 (67\%) had an accessory LV apical-basal muscle bundle identified on the preoperative CMR study. Among these $22 \mathrm{HCM}$ patients, the accessory LV apical-basal muscle bundle was identified and resected during 
the operation, judged by the surgeon to be contributing to the mechanism of LVOT obstruction in the following manner: (i) the apical portions of both the LV accessory apical-basal muscle bundle and anterolateral papillary muscle are often fused together; (ii) the anterolateral papillary muscle is positioned closer to the ventricular septum than normal, limiting its mobility during the cardiac cycle; (iii) the abnormal position of the papillary muscle tethers the mitral valve plane closer to the basal portion of the ventricular septum facilitating motion of the anterior leaflet toward the septum by the highvelocity ejection of blood (Figure 2).

\section{$\mathbf{G}+/ \mathbf{P}-$ family members}

Similar to HCM patients, the presence of an LV apical-basal muscle bundle identified by CMR was identified in nearly two-thirds of G+/P- family members (G+/P - subjects: $n=18$ of $30 ; 60 \%$ vs. HCM patients: $n=145$ of 230; 63\%; $P=0.9$ ) (Figure 3), which was significantly higher than in control subjects $(n=12$ of 126 ; $10 \% ; P<0.0001)$. Among those $G+/ P$ - family members with an $\mathrm{LV}$ apical-basal muscle bundle, MYBPC3 and MYH7 were the two most common genes identified (50 and $23 \%$, respectively). There was no difference with respect to the prevalence of LV apicalbasal muscle bundles between the two most common sarcomere genes involved in the disease process: MYBPC3: $n=10$ of 15 (67\%) vs. MYH7: $n=5$ of 7 (71\%); $P=0.95$.

\section{Reproducibility of left ventricular apical-basal muscle bundle}

Interobserver variability showed excellent agreement in the identification of LV apical-septal muscle bundle between the two observers $(\kappa=0.937)$. Similarly, intraobserver variability also showed excellent agreement for identification of LV apical-basal muscle bundle $(\kappa=0.869)$.

\section{Discussion}

Over the last decade, CMR has emerged as a particularly powerful imaging technique to characterize the diverse phenotypic expression of this genetic heart disease, ${ }^{4,7-10,21}$ leading to improved diagnostic and management strategies. Since performing CMR, we have observed an unusual accessory LV muscle structure extending from the apex to the basal anterior septum or basal anterior wall (traversing the LV cavity) in patients with HCM. Therefore, we felt it was timely to assemble a large cohort of HCM patients to assess the prevalence and clinical significance of this LV apical-basal muscle structure among a large cohort of genotyped HCM patients.

An LV apical-basal muscle bundle was present in the majority of HCM patients, indicating that this distinct morphologic structure of the LV myocardium appears to be part of the phenotypic expression of this disease. Left ventricular apical muscle bundles proved to be

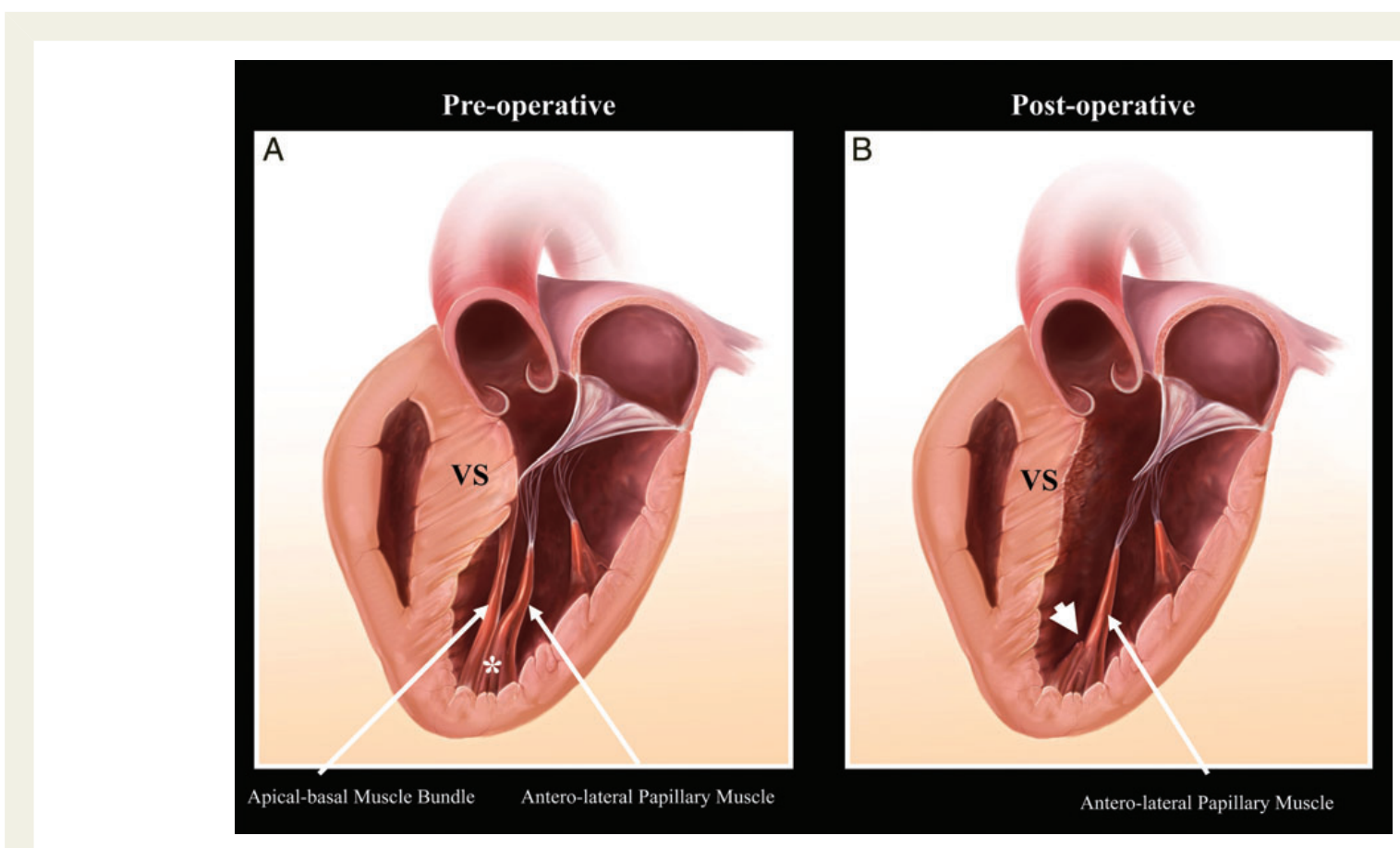

Figure 2 Artistic depiction of the contribution of left ventricular apical-basal muscle bundles to the generation of left ventricular outflow tract obstruction. (A) Situation prior to surgical myectomy: (i) accessory left ventricular apical-basal muscle bundle and the anterolateral papillary muscle are often fused together (at the apical portion $\left(^{*}\right)$ ), positioning the anterolateral papillary muscle closer to the ventricular septum than normal and limiting its mobility away from the septum during systole; (ii) this results in the mitral valve plane oriented in close proximity to the ventricular septum, facilitating motion of the anterior leaflet toward the septum by the high-velocity ejection of blood. (B) Situation after surgical myectomy: by completely excising the accessory muscle bundle, these abnormal muscular connections are eliminated, which ultimately result in a normal orientation of the mitral valve plane (i.e. and the leaflets coapting farther away from the basal ventricular septum). 

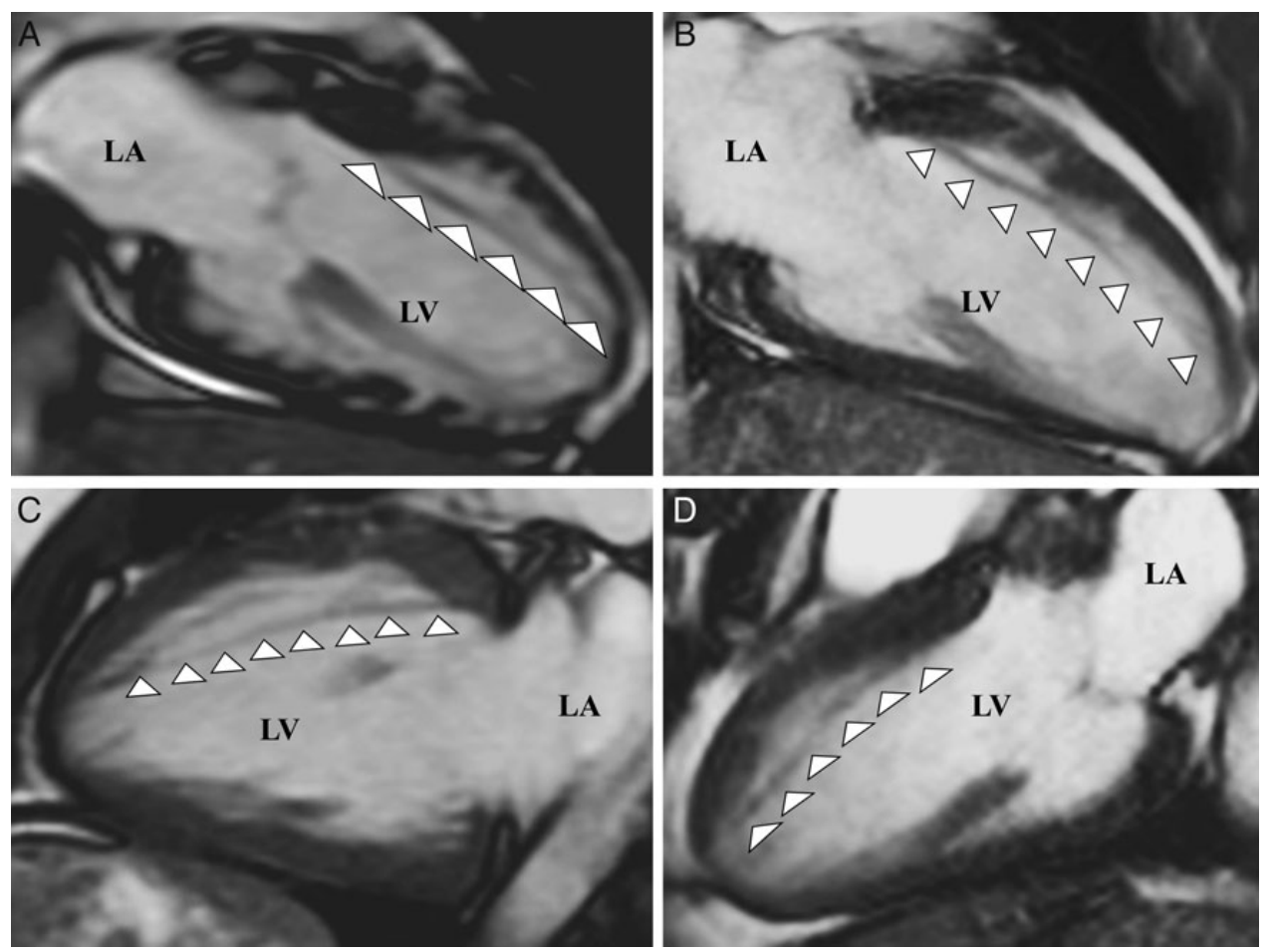

Figure 3 Spectrum of accessory left ventricular apical-basal muscle bundles in four G+/P- family members. (A) 27-year-old woman with a normal maximum left ventricular thickness of $11 \mathrm{~mm}$, and a disease-causing sarcomere protein gene mutation (MYBPC 3 c.927-9G $>A$, intron 11). (B) 28-year-old man with a normal maximum left ventricular thickness of $9 \mathrm{~mm}$, and a disease-causing MYBPC3 gene mutation (p.Glu728X). (C) 19-year-old man with a normal maximum left ventricular thickness of $9 \mathrm{~mm}$, and a disease-causing sarcomere protein gene mutation (MYH7 p.Gly741.Trp). (D) 42-year-old woman with a normal maximum left ventricular thickness of $12 \mathrm{~mm}$, and a disease-causing MYBPC3 mutation (p.Arg502Trp). Ao, aorta; LA, left atrium; LV, left ventricle.

independent of a number of demographic and clinical variables relevant to HCM disease expression, including maximal LV wall thickness. Indeed, HCM patients with more limited expressions of $L V$ wall thickening were equally as likely to have an LV apical-basal muscle bundle compared with those patients with substantial LV hypertrophy. The high prevalence of LV apical-basal muscle bundles among G+/Pfamily members (i.e. 60\%) underscores the important principle that these muscle bundles, in the absence of LV hypertrophy, are a potential CMR morphologic marker of genotype-positive status in family members. Furthermore, this muscle structure was also observed across the broad spectrum of ages, including a substantial proportion prior to adulthood.

Our current observations, particularly the finding that muscle bundles occur in the absence of hypertrophy in G+/P- family members and are present in young pre-adolescent HCM patients, suggest that these unique morphologic structures likely represent a congenital malformation. Other recent CMR-based observations in patients with HCM have demonstrated a number of morphologic abnormalities present early in development and independent of LV hypertrophy, including anomalous insertion of the anterolateral papillary muscle, ${ }^{7}$ elongated mitral valve leaflets, ${ }^{4,5}$ and myocardial crypts. $^{8-10}$ In total, these observations support the emerging principle that a number of morphologic abnormalities in HCM are likely present from birth, unlike LV hypertrophy which typically develops during adolescence as a result of the functional consequences caused by mutations encoding proteins of the cardiac sarcomere.

However, we wish to be cautious in promoting LV apical-basal muscle bundles as a specific finding in HCM, particularly since other populations with cardiovascular disease have not been systematically studied with CMR for the purposes of assessing LV apicalbasal muscle bundles. Nevertheless, in patients with equivocal LV wall thickening and/or family history of HCM, and in whom diagnosis remains ambiguous, the presence of an LV apical-basal muscle bundle can provide weight toward clarifying a clinical diagnosis of HCM.

Moreover, the observation that LV apical-basal muscle bundles were also identified in the majority of $\mathrm{G}+/ \mathrm{P}-$ family members provides further support that this unique morphologic feature likely represents an independent and primary component of HCM disease expression and can be considered a potential imaging marker for genetically affected status in HCM family members in the absence of the traditional phenotype (i.e. LV hypertrophy). In this regard, LV apical-basal muscle bundles may alone or in combination with a variety of other structural alterations previously identified in HCM family members who carry a disease-causing sarcomere mutation (i.e. elongated mitral valve leaflets, ${ }^{4,5}$ myocardial crypts, ${ }^{8-10}$ LGE, $^{22}$ serum biomarkers of myocardial fibrosis, ${ }^{23}$ and echocardiographic indices of diastolic dysfunction ${ }^{24,25}$ ) provide a provisional HCM diagnosis as a gene-positive carrier, thereby 
prompting strong consideration for obtaining a confirmatory diagnosis with genetic testing. ${ }^{16}$

The presence of an accessory LV apical-basal muscle bundle may also have implications for clinical management strategies. In the majority of patients undergoing surgical myectomy for relief of LVOT obstruction, an LV apical-basal muscle bundle was identified by the surgeon intraoperatively as a contributing factor to the mechanism of LVOT obstruction, and as a result completely resected during the operation. By operative excision of the accessory muscle bundles, the abnormal muscular connections were eliminated, ultimately resulting in a normal orientation of the mitral valve plane (i.e. with the leaflets coapting farther away from the basal ventricular septum). ${ }^{26,27}$ Therefore, by reliably identifying LV apical-basal muscle bundles, as well as other abnormalities of the mitral valve and subvalvular structure which can also contribute to LVOT obstruction (i.e. elongated mitral valve leaflets, accessory and apically placed papillary muscles, and anomalous insertion of the papillary muscle), preoperative CMR can provide important morphologic data to aid in surgical planning and strategy.

In this study, we used CMR to systematically evaluate for the presence of LV apical-basal muscles bundles in patients with HCM, as it has been our experience that 2D transthoracic echocardiography is often not capable of reliably detecting this particular structural abnormality. Indeed, LV apical-basal muscle bundles have largely gone unrecognized until the emergence of CMR, due to the fact that this narrow, thin structure is often confined predominantly to the distal portion of the LV chamber. This is similar to other observations with CMR in which other morphologic abnormalities of the distal portion of the LV chamber may not be well seen with echocardiography including focal hypertrophy confined to the apex or apical aneurysms. The unique imaging strengths of CMR, with its high spatial resolution and ability to generate images with sharp contrast between bright blood pool and dark myocardium, is particularly well suited to reliably identify this muscle structure.

\section{Limitations}

The present HCM cohort was assembled in a consecutive fashion, but with enrolment defined by eligibility for CMR. Therefore, a certain degree of preference with respect to patient selection was unavoidable within our study design. In addition, the size of our control population was limited and may not be completely representative of the general population. These features of our study design and patient selection may influence the generalizability of these data to the broader HCM patient population.

In conclusion, these CMR-based observations demonstrate that LV apical-basal muscle bundles are commonly identified in HCM patients and genotype-positive/phenotype-negative members and may represent a primary morphologic feature which further expands our appreciation for the diverse phenotypic expression of this genetic heart disease. The presence of an LV apical-basal muscle bundle can prompt confirmatory genetic testing in family members without LV hypertrophy and when identified preoperatively, may alter surgical planning.

\section{Funding}

H.R. receives funding from the Halpern HCM Research Fund. B.J.M. is a consultant for GeneDx. The other authors have no conflicts of interest to disclose. None of the disclosures pertain to this study and none of the funds/companies provided financial support for this study.

\section{Conflict of interest: none declared.}

\section{References}

1. Maron MS, Maron BJ, Harrigan C, Buros J, Gibson CM, Olivotto I, Biller L, Lesser JR, Udelson JE, Manning WJ, Appelbaum E. Hypertrophic cardiomyopathy phenotype revisited after 50 years with cardiovascular magnetic resonance. J Am Coll Cardiol 2009;54:220-228.

2. Olivotto I, Maron MS, Autore C, Lesser JR, Rega L, Casolo G, De Santis M, Quarta G, Nistri S, Cecchi F, Salton CJ, Udelson JE, Manning WJ, Maron BJ. Assessment and significance of left ventricular mass by cardiovascular magnetic resonance in hypertrophic cardiomyopathy. J Am Coll Cardiol 2008;52:559-566.

3. Kim DH, Handschumacher MD, Levine RA, Choi YS, Kim YJ, Yun SC, Song JM, Kang DH, Song JK. In vivo measurement of mitral leaflet surface area and subvalvular geometry in patients with asymmetrical septal hypertrophy: insights into the mechanism of outflow tract obstruction. Circulation 2010;122:1298-1307.

4. Maron MS, Olivotto I, Harrigan C, Appelbaum E, Gibson CM, Lesser JR, Haas TS, Udelson JE, Manning WJ, Maron BJ. Mitral valve abnormalities identified by cardiovascular magnetic resonance represent a primary phenotypic expression of hypertrophic cardiomyopathy. Circulation 2011;124:40-47.

5. Cavalcante JL, Barboza JS, Lever HM. Diversity of mitral valve abnormalities in obstructive hypertrophic cardiomyopathy. Prog Cardiovasc Dis 2012;54:517-522.

6. Maron MS, Hauser TH, Dubrow E, Horst TA, Kissinger KV, Udelson JE, Manning WJ. Right ventricular involvement in hypertrophic cardiomyopathy. Am J Cardiol 2007; 100:1293-1298.

7. Harrigan CJ, Appelbaum E, Maron BJ, Buros JL, Gibson CM, Lesser JR, Udelson JE, Manning WJ, Maron MS. Significance of papillary muscle abnormalities identified by cardiovascular magnetic resonance in hypertrophic cardiomyopathy. Am J Cardiol 2008;101:668-673.

8. Germans T, Wilde AA, Dijkmans PA, Chai W, Kamp O, Pinto YM, van Rossum AC. Structural abnormalities of the inferoseptal left ventricular wall detected by cardiac magnetic resonance imaging in carriers of hypertrophic cardiomyopathy mutations. J Am Coll Cardiol 2006;48:2518-2523.

9. Brouwer WP, Germans T, Head MC, van der Velden J, Heymans MW, Christiaans I, Houweling AC, Wilde AA, van Rossum AC. Multiple myocardial crypts on modified long-axis view are a specific finding in pre-hypertrophic HCM mutation carriers. Eur Heart J Cardiovasc Imaging 2012;13:292-297.

10. Maron MS, Rowin EJ, Lin D, Appelbaum E, Chan RH, Gibson CM, Lesser JR, Lindberg J, Haas TS, Udelson JE, Manning WJ, Maron BJ. Prevalence and clinical profile of myocardial crypts in hypertrophic cardiomyopathy. Circ Cardiovasc Imaging 2012;5:441-447.

11. Bruder O, Wagner A, Jensen CJ, Schneider S, Ong P, Kispert EM, Nassenstein K, Schlosser T, Sabin GV, Sechtem U, Mahrholdt H. Myocardial scar visualized by cardiovascular magnetic resonance imaging predicts major adverse events in patients with hypertrophic cardiomyopathy. J Am Coll Cardiol 2010;56:875-887.

12. Rubinshtein R, Glockner JF, Ommen SR, Araoz PA, Ackerman MJ, Sorajja P, Bos JM, Tajik AJ, Valeti US, Nishimura RA, Gersh BJ. Characteristics and clinical significance of late gadolinium enhancement by contrast-enhanced magnetic resonance imaging in patients with hypertrophic cardiomyopathy. Circ Heart Fail 2010;3:51-58.

13. O'Hanlon R, Grasso A, Roughton M, Moon JC, Clark S, Wage R, Webb J, Kulkarni M, Dawson D, Sulaibeekh L, Chandrasekaran B, Bucciarelli-Ducci C, Pasquale F, Cowie MR, McKenna WJ, Sheppard MN, Elliott PM, Pennell DJ, Prasad SK. Prognostic significance of myocardial fibrosis in hypertrophic cardiomyopathy. J Am Coll Cardiol 2010;56:867-874.

14. Adabag AS, Maron BJ, Appelbaum E, Harrigan CJ, Buros JL, Gibson CM, Lesser JR, Hanna CA, Udelson JE, Manning WJ, Maron MS. Occurrence and frequency of arrhythmias in hypertrophic cardiomyopathy in relation to delayed enhancement on cardiovascular magnetic resonance. J Am Coll Cardiol 2008;51:1369-1374.

15. MoonJC, McKenna WJ, McCrohonJA, Elliott PM, Smith GC, Pennell DJ. Toward clinical risk assessment in hypertrophic cardiomyopathy with gadolinium cardiovascular magnetic resonance. J Am Coll Cardiol 2003;41:1561-1567.

16. Gersh BJ, Maron BJ, Bonow RO, Dearani JA, Fifer MA, Link MS, Naidu SS, Nishimura RA, Ommen SR, Rakowski H, Seidman CE, Towbin JA, Udelson JE, Yancy CW. 2011 ACCF/AHA guideline for the diagnosis and treatment of hypertrophic cardiomyopathy: a report of the American College of Cardiology Foundation/American Heart Association Task Force on Practice Guidelines. Circulation 2011;124:e783-e831.

17. Klues HG, Roberts WC, Maron BJ. Anomalous insertion of papillary muscle directly into anterior mitral leaflet in hypertrophic cardiomyopathy. Significance in producing left ventricular outflow obstruction. Circulation 1991;84:1188-1197.

18. Rowin EJ, Maron BJ, Lesser JR, Rastegar H, Maron MS. Papillary muscle insertion directly into the anterior mitral leaflet in hypertrophic cardiomyopathy, its 
identification and cause of outflow obstruction by cardiac magnetic resonance imaging, and its surgical management. Am J Cardiol 2013;111:1677-1679.

19. Harrigan CJ, Peters DC, Gibson CM, Maron BJ, Manning WJ, Maron MS, Appelbaum E. Hypertrophic cardiomyopathy: quantification of late gadolinium enhancement with contrast-enhanced cardiovascular MR imaging. Radiology 2011;258:128-133.

20. Maron BJ, Maron MS, Semsarian C. Genetics of hypertrophic cardiomyopathy after 20 years: clinical perspectives. J Am Coll Cardiol 2012;60:705-715.

21. Moon JC, Fisher NG, McKenna WJ, Pennell DJ. Detection of apical hypertrophic cardiomyopathy by cardiovascular magnetic resonance in patients with non-diagnostic echocardiography. Heart 2004;90:645-649.

22. Rowin EJ, Maron MS, Lesser JR, Maron BJ. CMR with late gadolinium enhancement in genotype positive-phenotype negative hypertrophic cardiomyopathy. JACC Cardiovasc Imaging 2012;5:119-122.

23. Ho CY, Lopez B, Coelho-Filho OR, Lakdawala NK, Cirino AL, Jarolim P, Kwong R, Gonzalez A, Colan SD, Seidman JG, Diez J, Seidman CE. Myocardial fibrosis as an early manifestation of hypertrophic cardiomyopathy. N Engl J Med 2010;363: $552-563$.

24. Cardim N, Perrot A, Ferreira T, Pereira A, Osterziel KJ, Reis RP, Correia JF. Usefulness of Doppler myocardial imaging for identification of mutation carriers of familial hypertrophic cardiomyopathy. Am J Cardiol 2002;90:128-132.

25. De S, Borowski AG, Wang H, Nye L, Xin B, Thomas JD, Tang WH. Subclinical echocardiographic abnormalities in phenotype-negative carriers of myosin-binding protein C3 gene mutation for hypertrophic cardiomyopathy. Am Heart J 2011; 162:262-267 e263.

26. Kwon DH, Smedira NG, Thamilarasan M, Lytle BW, Lever H, Desai MY. Characteristics and surgical outcomes of symptomatic patients with hypertrophic cardiomyopathy with abnormal papillary muscle morphology undergoing papillary muscle reorientation. J Thorac Cardiovasc Surg 2010;140:317-324.

27. Dearani JA, Ommen SR, Gersh BJ, Schaff HV, Danielson GK. Surgery insight: septal myectomy for obstructive hypertrophic cardiomyopathy - the Mayo Clinic experience. Nat Clin Pract Cardiovasc Med 2007;4:503-512.

\section{CARDIOVASCULAR FLASHLIGHT}

\section{Transapical myectomy for severe mid-ventricular obstructive hypertrophic cardiomyopathy}

\section{Kevin C. Ong ${ }^{1}$, Jeffrey B. Geske ${ }^{1}$, Hartzell V. Schaff ${ }^{2}$, and Rick A. Nishimura ${ }^{1 *}$}

${ }^{1}$ Division of Cardiovascular Diseases, Department of Internal Medicine, Mayo Clinic, Gonda 5, 200 First Street SW, Rochester, MN 55905, USA and ${ }^{2}$ Division of Cardiovascular Surgery, Mayo Clinic, Rochester, MN, USA

* Corresponding author. Tel: +1 5072848342, Fax: +1 5072667929, Email: rnishimura@mayo.edu

An 83-year-old woman with hypertrophic cardiomyopathy was referred with progressive exertional dyspnoea. She had marked apical and mid-ventricular hypertrophy with mid-ventricular obstruction and a small apical aneurysm (Panels A1-3, Supplementary material online, Video S1). Doppler echocardiography through the left ventricle (Panel B1) identified an early peak systolic gradient of $36 \mathrm{mmHg}$ (Panel B1), mid-systolic cessation of flow (Panel B2), followed by re-emergence of forward flow in late systole and continuing into early diastole (paradoxical flow) (Panel B3). Cardiac catheterization demonstrated a $184 \mathrm{mmHg}$ mid-ventricular gradient (Panel D), pulmonary artery wedge pressure of $32 \mathrm{mmHg}$, and normal coronaries. She underwent apical and mid-ventricular myectomy (Panels C1-3) which resulted in symptom improvement.

Flow through an area of obstruction is required in order to derive a Doppler pressure gradient. In our patient, complete mid-ventricular obstruction led to mid-systolic flow cessation through the left ven-
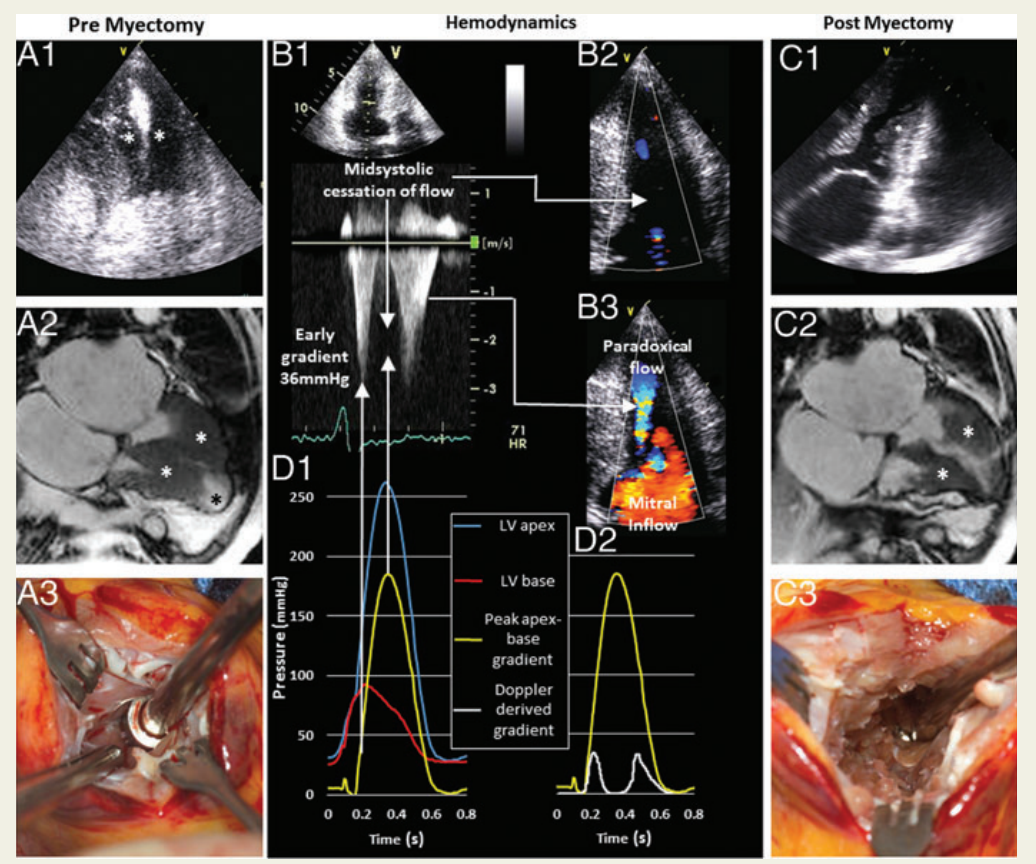

tricular cavity and the absence of a Doppler signal to determine a pressure gradient. Therefore, the true peak mid-ventricular pressure gradient recorded on haemodynamic catheterization occurred during apical mid-systolic isovolumic contraction when cavity obliteration prevented ejection of flow from the apex (Panels B1 and D1-2). This supports the concept that significant elevations in apical intracavitary pressure due to severe mid-ventricular obstruction may contribute to the genesis of an apical aneurysm. Therefore, non-invasive Doppler echocardiography may significantly underestimate the magnitude of mid-ventricular obstruction. Mid-ventricular myectomy relieves midventricular obstruction, and, in patients with significant secondary elevations in pulmonary artery wedge pressure, improves diastolic filling.

The authors have no potential conflicts of interest in regards to the contents of this manuscript.

Supplementary material is available at European Heart Journal online.

Published on behalf of the European Society of Cardiology. All rights reserved. (C) The Author 2014. For permissions please email: journals.permissions@oup.com 